PROCEEDINGS OF THE

AMERICAN MATHEMATICAL SOCIETY

Volume 133, Number 12, Pages 3497-3503

S 0002-9939(05)07891-3

Article electronically published on July 13, 2005

\title{
LORENTZ SPACE EXTENSION OF STRICHARTZ ESTIMATES
}

\author{
CHEONGHEE AHN AND YONGGEUN CHO
}

(Communicated by Christopher D. Sogge)

\begin{abstract}
In this paper, Strichartz estimates for the solution of the Schrödinger evolution equation are considered on a mixed normed space with Lorentz norm with respect to the time variable.
\end{abstract}

\section{INTRODUCTION}

We consider Strichartz estimates on the unitary group $U(t)=e^{-i t \Delta}$ on $\mathbb{R}^{n}$ $(n \geq 3)$. As usual, Strichartz estimates on this group can be formulated on the Lebesgue space as follows [10]:

$$
\begin{gathered}
\|U(t) f\|_{L^{q} L^{r}} \leq C\|f\|_{L^{2}}, \\
\left\|\int_{0}^{t} U(t-s) F(s) d s\right\|_{L^{q} L^{r}} \leq C\|F\|_{L^{\tilde{q}^{\prime}} L^{\tilde{r}^{\prime}}}
\end{gathered}
$$

for any $f \in L^{2}$ and $F \in L^{\tilde{q}^{\prime}} L^{\tilde{r}^{\prime}}$, provided $\frac{1}{q}+\frac{n}{2 r}=\frac{n}{4}, \frac{1}{\tilde{q}}+\frac{n}{2 \tilde{r}}=\frac{n}{4}$ and $2 \leq q, \tilde{q}, r, \tilde{r} \leq$ $\infty$. Such a pair $(q, r)$ is called admissible. Here we denote by $L^{q} L^{r}$ a Banach space defined by the mixed spacetime norm $\|u\|_{L^{q} L^{r}}=\left(\int_{0}^{\infty}\left(\int_{\mathbb{R}^{n}}|u(x, t)|^{r} d x\right)^{\frac{q}{r}}\right)^{\frac{1}{q}}$.

The purpose of this paper is to extend the known Strichartz estimates on $L^{q} L^{r}$ to the one on the Lorentz space $L^{q, s} L^{r}$ with $s \in[2, q]$. The Lorentz space $L^{q, s}$ is defined by all measurable functions $g$ satisfying

$$
\|g\|_{L^{q, s}}=\left(\frac{s}{q} \int_{0}^{\infty}\left[t^{\frac{1}{q}} g^{*}(t)\right]^{s} \frac{d t}{t}\right)^{\frac{1}{s}}<\infty
$$

where $g^{*}$ is the decreasing rearrangement of $g$ (see [1] and 20]). From the dispersion of $U(t)$ such that $\|U(t) f\|_{L^{r}} \leq C t^{-n(1 / 2-1 / r)}\|f\|_{L^{r^{\prime}}}$ for any $r \in[2, \infty]$ and the fact that $t^{-n(1 / 2-1 / r)} \in L^{\frac{2 r}{n(r-2)}, \infty}$ for any $r \in\left[2, \frac{2 n}{n-2}\right]$, one can easily show that for any admissible pair $(q, r)$ with $q>2$,

$$
\begin{gathered}
\|U(t) f\|_{L^{q, 2} L^{r}} \leq C\|f\|_{L^{2}}, \\
\left\|\int_{0}^{t} U(t-s) F(s) d s\right\|_{L^{q, 2} L^{r} \cap L^{\infty} L^{2}} \leq C\|F\|_{L^{q^{\prime}, 2} L^{r^{\prime}}}
\end{gathered}
$$

Received by the editors February 16, 2004 and, in revised form, July 5, 2004.

2000 Mathematics Subject Classification. Primary 35J10, 42B25.

Key words and phrases. Strichartz estimate, Schrödinger equation, Lorentz space.

The first author was supported by KOSEF R01-2004-000-10072-0 and the second author by Korea Research Foundation KRF-2003-005-C00011. 
For the details of the proof, see [12. But in the case when $U(t)=e^{i t H}$, where $H=-\Delta+V$, we cannot apply this argument to $U(t)$ because in general it is hard to obtain the dispersion (time decay) as above. Moreover, to deal with nonlinear problems, we need many more admissible pairs $(q, r)$ than in the second inequality of (1.1). To settle these situations, we use a boundedness of Lorentz space version for a low diagonal operator, which is an integral version of the maximal operator for filtration (see 4,5$]$ ). The boundedness on Lebesgue space was studied by T. Tao 21 and H. Smith and C. Sogge [19.

In Section 2, we prove the boundedness of a low diagonal operator on Lorentz space. Exploiting this, in Section 3 we prove the Strichartz estimate on $U(t)$ and then as its consequence we treat the case when a nonlinear potential term or a long-range linear potential $V$ is involved.

Throughout the paper we use the notation $A \lesssim B$ and $A \sim B$ to denote

$$
|A| \leq C B \quad \text { and } \quad C^{-1} B \leq|A| \leq C B \text { respectively, }
$$

where $C$ is a generic constant varying line by line and depending only on $n$ and admissible pairs.

\section{LOW DIAGONAL OPERATOR}

Let $\mathcal{A}$ and $\mathcal{B}$ be Banach spaces and let $T$ be an integral operator $L^{p, r}(0, \infty ; \mathcal{A}) \rightarrow$ $L^{q, s}(0, \infty ; \mathcal{B})$ for some $p, r, q, s$ with a kernel $k(t, s)$ such that

$$
T F(t)=\int_{0}^{\infty} k(t, s) F(s) d s
$$

Then the low diagonal operator $\tilde{T}$ is defined by

$$
\tilde{T} F(t)=\int_{0}^{t} k(t, s) F(s) d s .
$$

We have the following result.

Proposition 2.1. If $1 \leq p \leq r<s \leq q<\infty$, then we have

$$
\|\tilde{T} F\|_{L^{q, s}(0, \infty ; \mathcal{B})} \lesssim\|F\|_{L^{p, r}(0, \infty ; \mathcal{A})} .
$$

If $p=r, q=s$ and $p<q$, then it is shown in [19] or [21] that $\tilde{T}$ is bounded from $L^{p}(0, \infty ; \mathcal{A})$ to $L^{q}(0, \infty ; \mathcal{B})$ and its norm satisfies $\|\tilde{T}\| \leq C\|T\|$. One can find in [4, 5] that a similar result on the maximal operator $T^{*}(f)=\sup _{n}\left|T\left(f \chi_{E_{n}}\right)\right|\left(E_{n} \subset\right.$ $\left.E_{n+1}\right)$ does hold for a known linear operator $T$ bounded from $L^{p, r}$ to $L^{q, s}$.

To prove Proposition 2.1, we use the following lemma.

Lemma 2.2 (Lemma 2.1 in [4]). Let $I, J$ be disjoint measurable subsets in a measure space $X$ and let $f, g$ be measurable functions on $X$. Then for any pair $(q, s)$ with $1 \leq s \leq q<\infty$, we have

$$
\left\|f \chi_{I}+g \chi_{J}\right\|_{L^{q, s}}^{s} \leq\left\|f \chi_{I}\right\|_{L^{q, s}}^{s}+\left\|g \chi_{J}\right\|_{L^{q, s}}^{s},
$$

and for any pair $(p, r)$ with $1 \leq p \leq r$, we also have

$$
\left\|f \chi_{I}+g \chi_{J}\right\|_{L^{p, r}}^{r} \geq\left\|f \chi_{I}\right\|_{L^{p, r}}^{r}+\left\|g \chi_{J}\right\|_{L^{p, r}}^{r} .
$$


Proof of Proposition 2.1. For simplicity we denote $\|F\|_{L^{p, r}(0, \infty ; \mathcal{A})}$ by $\|F\|_{L_{\mathcal{A}}^{p_{\mathcal{A}} r} \text {. }}$ Without loss of generality, we may assume that $\|F\|_{L_{\mathcal{A}}^{p, r}}=1$. Then using Lemma 2.2. we can find mutually disjoint subsets $B_{k}^{l}$ of $[0, \infty)$ such that

(1) $\bigcup_{k=1}^{2^{l}} B_{k}^{l}=[0, \infty)$ and $\left\|\chi_{B_{k}^{l}} F\right\|_{L_{\mathcal{A}}^{p, r}}^{r} \leq 2^{-l}$ for all $k, l$,

(2) $B_{k}^{l}$ lies entirely to the left of $B_{i}^{l}$ if $k<i$.

For the existence of such sets, see 4 and $[\underline{6}$. From the diagonal decomposition as in the proof of Lemma 4.2 in [6], one can easily deduce that

$$
\begin{aligned}
\chi_{\mathbb{R}^{+}}(t-s)\|F(s)\|_{\mathcal{A}}\|F(t)\|_{\mathcal{A}} \\
\quad=\left(\sum_{l=1}^{\infty} \sum_{k=1, k \text { odd }}^{2^{l}} \chi_{B_{k}^{l}}(s) \chi_{B_{k+1}^{l}}(t)\right)\|F(s)\|_{\mathcal{A}}\|F(t)\|_{\mathcal{A}} .
\end{aligned}
$$

Recall $\tilde{T} F(t)=\int_{0}^{t} k(t, s) F(s) d s$ and, substituting the above identity into $\tilde{T}(F)$, we have

$$
\begin{aligned}
\tilde{T} F(t) & =\sum_{l=1}^{\infty} \sum_{k=1, k \text { odd }}^{2^{l}} \chi_{B_{k+1}^{l}}(t) \int_{0}^{\infty} k(t, s) F(s) \chi_{B_{k}^{l}}(s) d s \\
& =\sum_{l=1}^{\infty} \sum_{k=1, k \text { odd }}^{2^{l}} \chi_{B_{k+1}^{l}}(t) T\left(\chi_{B_{k}^{l}} F\right)(t) .
\end{aligned}
$$

Since the sets $B_{k+1}^{l}$ are mutually disjoint, we deduce from Lemma 2.2 that

$$
\|\tilde{T} F\|_{L_{\mathcal{B}}^{q, s}} \leq \sum_{l}\left\|\sum_{k}^{\prime} \chi_{B_{k+1}^{l}}(\cdot) T\left(\chi_{B_{k}^{l}} F\right)\right\|_{L_{\mathcal{B}}^{q, s}}
$$

where $\sum_{k}^{\prime}$ is the sum over odd $k$.

Now fix $l$ and note that $q \geq s$ and $B_{k+1}^{l}$ are disjoint. By (2.2) in Lemma 2.2, we have the following:

$$
\begin{aligned}
\left\|\sum_{k}^{\prime} \chi_{B_{k+1}^{l}} T\left(F \chi_{B_{k}^{l}}\right)\right\|_{L_{\mathcal{B}}^{q, s}}^{s} & \leq \sum_{k}^{\prime}\left\|\chi_{B_{k+1}^{l}} T\left(F \chi_{B_{k}^{l}}\right)\right\|_{L_{\mathcal{B}}^{q, s}}^{s} \\
& \leq \sum_{k}^{\prime}\left(\|T\|_{L_{\mathcal{A}}^{p, r} \rightarrow L_{\mathcal{B}}^{q, r}}\right)^{s}\left\|F \chi_{B_{k}^{l}}\right\|_{L_{\mathcal{A}}^{p, r}}^{s}
\end{aligned}
$$

The last term in the above inequality is bounded by

$$
\sum_{k}^{\prime}\left(\|T\|_{\left.L_{\mathcal{A}}^{p, r} \rightarrow L_{\mathcal{B}}^{q, r}\right)}\right)^{s} 2^{-\left(\frac{s}{r}-1\right) l}\left\|F \chi_{B_{k}^{l}}\right\|_{L_{\mathcal{A}}^{p, r}}^{r} .
$$

Since $p \leq r$ and for each $l, B_{k}^{l}$ are disjoint, from the inequality (2.3), we deduce that $\sum_{k}^{\prime}\left\|F \chi_{B_{k}^{l}}\right\|_{L_{\mathcal{A}}^{p, r}}^{r} \leq\|F\|_{L_{\mathcal{A}}^{p_{j} r}}^{r}=1$, which implies the proposition. 


\section{Strichartz estimate}

We first consider the solution $u(t)=U(t) f=e^{-i t \Delta} f$ to the initial value problem

$$
\left\{\begin{array}{l}
i \partial_{t} u(x, t)-\Delta u(x, t)=0, \quad(x, t) \in \mathbb{R}^{n} \times \mathbb{R}^{1}, \\
u(x, 0)=f(x),
\end{array}\right.
$$

and we have the following.

Theorem 3.1. For any admissible pair $(q, r),(\tilde{q}, \tilde{r})$ and $s$ with $2<s \leq q$, we have

$$
\left\|\int_{0}^{t} U(t-s) F(s) d s\right\|_{L^{q, s} L^{r}} \lesssim\|F\|_{L^{\tilde{q}^{\prime}, 2} L^{\tilde{r}^{\prime}} \cap L^{2} L^{\frac{2 n}{n+2}, 2}} .
$$

Proof. For the proof of (3.2), let $k(t, s)=U(t-s), \mathcal{A}=L^{\tilde{r}^{\prime}}$ or $L^{\frac{2 n}{n+2}, 2}, \mathcal{B}=L^{r}$ and $T F=\int_{0}^{\infty} k(t, s) F(s) d s$. Then since $\tilde{q}^{\prime} \leq 2<s \leq q$, in view of Proposition 2.1] we have only to show that

$$
\left\|\int_{0}^{\infty} U(t-s) F(s) d s\right\|_{L^{q, s} L^{r}} \lesssim\|F\|_{L^{\tilde{q}^{\prime}, 2} L^{\tilde{r}^{\prime}} \cap L^{2} L^{\frac{2 n}{n+2}, 2}} .
$$

To show this, observe from (1.1) and $L^{q, s} \subset L^{q, 2}$ for all $s \geq 2$ (see [20]) that

$$
\begin{gathered}
\left\|\int_{0}^{\infty} U(t-s) F(s) d s\right\|_{L^{q, s} L^{r}}^{2} \lesssim\left\|\int_{0}^{\infty} U(-s) F(s) d s\right\|_{L^{2}}^{2} \\
=\int_{0}^{\infty} \int_{0}^{\infty}\langle U(-s) F(s), U(-\tau) F(\tau)\rangle d s d \tau .
\end{gathered}
$$

Then from the endpoint result of T. Tao and M. Keel [10, the right-hand side of (3.3) is bounded by $\|F\|_{L^{2} L^{\frac{2 n}{n+2}, 2}}^{2}$. The remaining part of the theorem can be obtained by the duality of Lorentz space $\left(L^{q, s}\right)^{\prime}=L^{q^{\prime}, s^{\prime}}$ and the second part of (1.1).

Remark 3.2. The estimate (3.2) cannot be derived directly by an interpolation between estimates in (1.1), because the complex (even real) interpolation of two Banach spaces with mixed norms requires a strong constraint among exponents in the norm. For instance, see [13, 8, 11].

Remark 3.3. The method in the proof of Theorem 3.1 can be applied directly to the wave equation and the Klein-Gordon equation. Moreover, we can apply it to the unitary group $U(t)=e^{-i t D^{a}}(a>0, a \neq 1)$, where $D^{a}=(-\Delta)^{\frac{a}{2}}$. In fact, using Littlewood-Paley theory and the Van der Corput lemma, we can easily see the following dispersive estimates:

$$
\begin{aligned}
&\|U(t) f\|_{L^{r}} \lesssim t^{-\frac{n}{a}\left(1-\frac{2}{r}\right)}\|f\|_{L^{r^{\prime}}} \quad \text { for } \quad a \geq 2 \\
&\|U(t) f\|_{L^{r}} \lesssim t^{\frac{n}{2}\left(1-\frac{2}{r}\right)}\|f\|_{\dot{W}^{\theta, r^{\prime}}} \quad \text { for } \quad \theta=\frac{n}{2}\left(1-\frac{2}{r}\right)(2-a), 0<a<2, a \neq 1,
\end{aligned}
$$

where $\dot{W}^{\theta, r^{\prime}}$ is the fractional Sobolev space. The first inequality can be seen in [16. Hence combining these and Proposition 2.1, we obtain similar results to (1.1) and (3.2) with pairs $(q, r)$ such that $\frac{1}{q}+\frac{n}{a r}=\frac{n}{2 a}$ with $q \geq 2$ if $2 \leq a \leq n$ and $q>\frac{2 a}{n}$ if $a>n$, and $\frac{1}{q}+\frac{n}{2 r}=\frac{n}{4}$ if $0<a<2$. In the case when $a<2$, the mixed norm $L^{q} L^{r}$ should be replaced by the Sobolev norm $L^{q} \dot{W}^{\theta, r}$. 
As an application of Theorem 3.1, we can derive Strichartz estimates of the solution to the nonlinear problem with $H^{1}$-critical nonlinearity:

$$
\left\{\begin{array}{l}
i \partial_{t} u-\Delta u+|u|^{\frac{4}{n-2}} u=0 \quad \text { in } \quad(x, t) \in \mathbb{R}^{n+1}, \\
u(0)=f \in H^{1} \text { in } \mathbb{R}^{n} .
\end{array}\right.
$$

Corollary 3.4. If the initial data is sufficiently small, then there exists a unique solution $u \in L^{q, s}\left(0, \infty ; W^{1, r}\right) \cap L^{2}\left(0, \infty ; W^{1, \frac{2 n}{n-2}}\right) \cap C\left([0, \infty) ; H^{1}\right)$ for any admissible pair $(q, r)$ with $q>2$ and $2<s \leq q$.

Proof of Corollary 3.4. Since the existence of a unique $H^{1}$-solution is well known (for details see [3]), it suffices to prove that $u \in L^{q, s}\left(0, \infty ; W^{1, r}\right)$. From Duhamel's principle, we deduce

$$
u(x, t)=U(t) f-i \int_{0}^{t} U(t-s)\left(|u|^{\frac{4}{n-2}} u\right) d s .
$$

Using (1.1) and (3.2), we have

$$
\|D u\|_{L^{q, s} L^{r}} \lesssim\|D f\|_{L^{2}}+\left\|D\left(|u|^{\frac{4}{n-2}} u\right)\right\|_{L^{\tilde{q}^{\prime}, 2} L^{\tilde{r}^{\prime}}} .
$$

We can always find an admissible pair $\left(q_{0}, r_{0}\right)$ with $r_{0}<n$ and $2<s_{0}<q_{0}$, and $(\tilde{q}, \tilde{r})$ and $1<\tilde{s}<2$ such that

$$
\frac{1}{\tilde{q}}=\frac{\frac{4}{n-2}}{q_{0}}+\frac{1}{q_{0}}, \quad \frac{1}{\tilde{r}}=\frac{\frac{4}{n-2}}{r_{0}^{*}}+\frac{1}{r_{0}}, \quad \frac{1}{\tilde{s}}=\frac{\frac{4}{n-2}}{s_{0}}+\frac{1}{s_{0}},
$$

where $r_{0}^{*}=\frac{n r_{0}}{n-r_{0}}$. Thus from Leibnitz' rule [7, 9], Hölder's inequality on Lorentz space [12, 15] and Sobolev embedding [1], we deduce that

$$
\|D u\|_{L^{q_{0}, s_{0}} L^{r_{0}}} \lesssim\|D f\|_{L^{2}}+\|D u\|_{L^{q_{0}, s_{0}} L^{r_{0}}}^{\frac{n+2}{n-2}} .
$$

Since $\|f\|_{H^{1}}$ is small, we have $\|D u\|_{L^{q_{0}}, s_{0} L^{r_{0}}} \lesssim\|D f\|_{L^{2}}$. Finally, since we can choose $(\tilde{q}, \tilde{r})$ arbitrarily to be admissible, for any admissible pair $(q, r)$ and $s$ with $q>2$ and $2<s \leq q$, we have

$$
\|D u\|_{L^{q, s} L^{r}} \lesssim\|D f\|_{L^{2}}+\|D u\|_{L^{q_{0}, s_{0} L^{r_{0}}}}^{\frac{n+2}{n-2}} \lesssim\|D f\|_{L^{2}}+\|D f\|_{L^{2}}^{\frac{n+2}{n-2}} .
$$

In a similar way, we can also derive from the smallness of $\|f\|_{H^{1}}$,

$$
\|u\|_{L^{q, s} L^{r}} \lesssim\|f\|_{L^{2}} .
$$

Another consequence of Theorem 3.1 is a Strichartz estimate on Lorentz space for the Schrödinger operator $H=-\Delta+V$ with potential $V$ satisfying

(1) $V \in C^{1}\left(\mathbb{R}^{n} \backslash\{0\}\right), \quad \sup _{\mathbb{R}^{n}}|x|^{2}|V(x)|<\infty$,

(2) the operator $-\Delta^{\prime}+|x|^{2} V+\frac{(n-2)^{2}}{4}$ is positive on every sphere,

(3) the operator $-\Delta^{\prime}+|x|^{2} \widetilde{V}+\frac{(n-2)^{2}}{4}$ is positive on every sphere,

where $\Delta^{\prime}$ is the spherical Laplacian and $\widetilde{V}=-\partial_{r}(r V(x))$. Under the above assumption, the operator $H$ becomes a positive, unique self-adjoint extension of $-\Delta+V$ and is defined as a form sum (see 2, 17]). Recently, N. Burq, F. Planchon, J. G. Stalker and A. S. Tahvildar-Zadeh [2] showed that if $u$ is a unique solution of the equation

$$
i u_{t}+H u=0, \quad u(0)=f
$$


with $V$ satisfying (1)-(3), then for any admissible pair $(q, r)$,

$$
\left\||x|^{-1} u\right\|_{L^{2} L^{2}}+\|u\|_{L^{q} L^{r}} \lesssim\|f\|_{L^{2}}
$$

holds. The Lorentz space version is the following:

Corollary 3.5. If $u$ is a unique solution of (3.5), then for any admissible pair $(q, r)$ and $2<s \leq q$, it follows that

$$
\|u\|_{L^{q, s} L^{r}} \lesssim\|f\|_{L^{2}} .
$$

Proof of Corollary 3.5. The solution $u$ can be written as $u(x, t)=U(t) f-$ $i \int_{0}^{t} U(t-s)(V u) d s$, where $U(t)=e^{-i t \Delta}$. From (1.1) and (3.2), we deduce that

$$
\|u\|_{L^{q, s} L^{r}} \lesssim\|f\|_{L^{2}}+\|V u\|_{L^{2} L^{\frac{2 n}{n+2}, 2}} .
$$

Now by (3.6) and Hölder's inequality on Lorentz space, we have

$$
\|V u\|_{L^{2} L^{\frac{2 n}{n+2}, 2}}=\left\||x| V|x|^{-1} u\right\|_{L^{2} L^{\frac{2 n}{n+2}, 2}} \lesssim\||x| V\|_{L^{n, \infty}}\left\||x|^{-1} u\right\|_{L^{2} L^{2}} \lesssim\|f\|_{L^{2}} .
$$

Remark 3.6. One can apply the low diagonal operator argument to the Schrödinger operator with finite rank, short-range perturbation or time dependent potential, and easily obtain a result similar to Corollary 3.5. For the Lebesgue space estimate, see 14] for finite rank perturbation, and [18] for short-range perturbation and for time dependent potential, respectively.

\section{REFERENCES}

[1] J. Bergh and J. Löfström, Interpolation Spaces, Springer, New York, (1976). MR0482275 $(58: 2349)$

[2] N. Burq, F. Planchon, J.G. Stalker and A. S. Tahvildar-Zadeh, Strichartz estimates for the Wave and Schrödinger Equations with Potentials of Critical Decay, in preprint.

[3] T. Cazenave and F. B. Weissler, Critical nonlinear Schrödinger equation, Nonlinear Anal. 14 (1990), 807-836. MR1055532 (91j:35252)

[4] Y. Cho, E. Koh and S. Lee, A maximal inequality for filtration on some function spaces, Osaka J. Math.41 (2004), 267-276. MR2069087

[5] M. Christ and A. Kiselev, Maximal functions associated to filtrations, J. Func. Anal. 179 (1998), 409-425. MR1809116 (2001i:47054)

[6] M. Christ and A. Kiselev, Absolutely continuous spectrum for one-dimensional Schrödinger operators with slowly decaying potentials: some optimal results, J. Amer. Math. Soc. 11 (1998), 771-797. MR1621882 (99g:34166)

[7] M. Christ and M. I. Weinstein, Dispersion of small amplitude solutions of the generalized Korteweg-de Vries equation, J. Func. Anal. 100 (1991), 87-109. MR1124294 (92h:35203)

[8] M. Cwikel, On $\left(L^{p_{0}}\left(A_{0}\right), L^{p_{1}}\left(A_{1}\right)\right)_{\theta, q}$, Proc. Amer. Math. Soc. 44 (1974), 286-292. MR0358326 (50:10792)

[9] T. Kato, On nonlinear Schrödinger equations II. $H^{s}$-solutions and unconditional wellposedness, Journal D'Analyse Mathématique, 67 (1995), 281-306. MR1383498 (98a:35124a)

[10] M. Keel and T. Tao, Endpoint Strichartz estimates, Amer. J. Math. 120 (1998), 955-980. MR1646048 (2000d:35018)

[11] J. L. Lions and J. Peetre, Sur une classe d'espaces d'interpolation, Inst. Hautes Études Sci. Publ. Math. 19 (1964), 5-68. MR0165343(29:2627)

[12] K. Nakanishi, Asymptotically-free solutions for the short-range nonlinear Schrödinger equation, SIAM J. Math. Anal. 32 (2001), 1265-1271. MR1856248 (2002g:35197)

[13] K. Nakanishi and T. Ozawa, Remarks on scattering for nonlinear Schrödinger equations, Nonlinear Differ. Equ. Appl. 9 (2002), 45-68. MR1891695 (2003a:35177)

[14] F. Nier and A. Soffer, Dispersion and Strichartz estimates for some fnite rank perturbations of the Laplace operator, J. Func. Anal. 198 (2003), 511-535. MR1964550 (2004m:47102) 
[15] R. O'Neil, Convolution operators and L(p,q) spaces, Duke Math. J. 30 (1963), 129-142. MR0146673 (26:4193)

[16] H. Pecher, $L^{p}$-Abschätzungen and klassische Lösungen für nichtlineare Wellengleichungen. I., Math. Z. 150 (1976), 159-183. MR0435604(55:8563a)

[17] M. Reed and B. Simon, Methods of modern mathematical physics, I-IV, Academic Press, New York-London, (1978). MR0493419|(58:12429a) MR0493420|(58:12429b) MR0493421 $(58: 12429 \mathrm{c})$

[18] I. Rodinianski and W. Schlag, Time decay for solutions of Schrödinger equations with rough and time-dependent potentials, Invent. Math. 155 (2004), no. 3, 451-513. MR2038194

[19] H. Smith and C. Sogge, Global Strichartz estimates for nontrapping perturbations of the Laplacian, Comm. Partial Differential Equations 25 (2000), 2171-2183. MR.1789924 $(2001 \mathrm{j}: 35180)$

[20] E. M. Stein and G. Weiss, Introduction to Fourier Analysis on Euclidean Spaces, Princeton Univ. Press, (1971). MR0304972 (46:4102)

[21] T. Tao, Spherically averaged endpoint Strichartz esimates for the two-dimensional Schrödinger equation, Comm. Partial Differential Equations 25 (2000), 1471-1485. MR:1765155 (2001h:35038)

Department of Mathematics, Yonsei University, Sinchon-dong, Seodaemun-gu, Seoul, Republic of Korea

E-mail address: purehope@yonsei.ac.kr

Department of Mathematics, Yonsei University, Sinchon-dong, Seodaemun-gu, Seoul, RePUBLIC OF Korea

E-mail address: bonobono@postech.ac.kr

Current address: Department of Mathematics, Hokkaido University, Sapporo 060-0810, Japan

E-mail address: ygcho@math.sci.hokudai.ac.jp 